\title{
New developments in reaction theory: preparing for the FRIB era
}

\author{
F.M. Nunes ${ }^{1,2, \star}$, P.C. Capel ${ }^{3,4}$, Ch. Elster ${ }^{5}$, L. Hlophe ${ }^{1}$, Jin Lei ${ }^{1}$, Weichuan Li ${ }^{1}$, A.E. Lovell ${ }^{1,2}$, G. \\ Potel $^{1}$, J. Rotureau ${ }^{1}$, and T. Poxon-Pearson ${ }^{1,2}$ \\ ${ }^{1}$ National Superconducting Cyclotron Laboratory, Michigan State University, East Lansing, MI 48824 \\ ${ }^{2}$ Department of Physics and Astronomy, Michigan State University, East Lansing, MI 48824-1321 \\ ${ }^{3}$ Physique Nucléaire et Physique Quantique, Université Libre de Bruxelles (ULB), B-1050 Brussels \\ ${ }^{4}$ Institut für Kernphysik, Technische Universität Darmstadt, 64289 Darmstadt, Germany \\ ${ }^{5}$ Institute of Nuclear and Particle Physics, and Department of Physics and Astronomy, Ohio University, \\ Athens, $\mathrm{OH} 45701$, USA
}

\begin{abstract}
This is a brief report on the progress made towards an exact theory for (d,p) on heavy nuclei, which is important to determine neutron capture rates for r-process nuclei. We first discuss the role of core excitation in the framework of Faddeev equations. Following that, we provide the status of the Faddeev theory being developed in the Coulomb basis with separable interactions. We then present some recent developments on nonlocal nucleon optical potentials. Finally, the progress on the theory transfer to the continuum is summarized.
\end{abstract}

\section{Introduction}

Since the early eighties, reaction experiments with radioactive beams have shed light on so many intriguing phenomena, from nuclear halos [1] to a strong dependence of the expected shell occupancy with the asymmetry of the system [2]. This is an exciting time for low energy nuclear physics because there are new facilities coming online worldwide, like the Facility for Rare Isotope Beams (FRIB), which will increase substantially the beam intensity and enable studies in unexplored regions of the nuclear chart, particularly toward neutron-rich heavy nuclei. Reaction probes are versatile and provide detailed information on many different nuclear properties ranging from shell structure, skins and pairing to the extraction of capture rates for the r-process and rp-process, as well as many applications such as stockpile stewardship. For example, neutron capture reactions on unstable nuclei cannot be measured directly. An attractive indirect method involves extracting this information from transfer $(d, p)$ reactions. The essential element in the analysis of these experiments is reaction theory: reaction theory is crucial to disentangle the desired structure or astrophysical information from experiments.

In this work, we provide an overview of a few recent developments that are taking place in the field of nuclear reactions, with focus on nucleon induced and deuteron induced reactions. In Section 2 we discuss recent results including core excitation in transfer (d,p) reactions [3]. Next, in Section 3, we present recent results for solving the Faddeev equations for (d,p) using separable interactions. We also discuss two different approaches to obtain the nucleon optical potentials (Section 4) and mention

^e-mail: nunes@nscl.msu.edu 


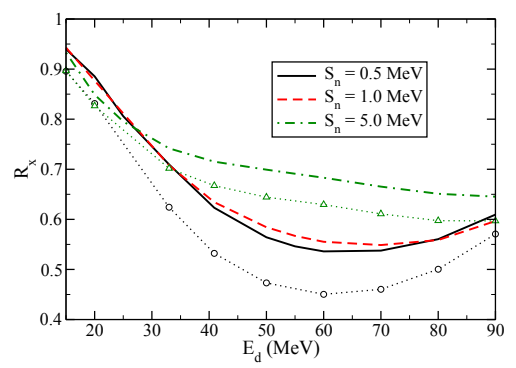

Figure 1. Spectroscopic factor ratio $R_{x}$ as a function of the beam energy extracted from ${ }^{10} \mathrm{Be}(\mathrm{d}, \mathrm{p}){ }^{11} \mathrm{Be}$ for various separation energies of the final nucleus. The lines with symbols are the results obtained when the excitation energy of ${ }^{10} \mathrm{Be}$ is strongly reduced. Figure from [3].

a recent review on inclusive (d,p) populating continuum states (Section 5). Finally, the conclusions are presented in Section 6.

\section{Core excitation in $(d, p)$ reactions}

Deuteron induced reactions on targets are most often cast as a three-body problem. Over the last decade, much work has gone into establishing the methods used to solve the three-body scattering problem. Examples are the benchmarks of the adiabatic wave approximation method and the continuum discretized coupled-channel method against the exact Faddeev approach for transfer reactions $[4,5]$. In those studies, the system was treated as $n+p+A$, and no excitation was allowed for $A$. When is this approximation adequate?

Core-excitation effects have been studied in detail in the context of the breakup of loosely bound two-body-like projectiles (e.g. [6]). Results from these breakup studies demonstrate that, when including core excitation dynamically in the reaction, observables are significantly modified. Until recently, no equivalent work using non-perturbative approaches has been done for the transfer channel.

A couple of years ago, the full Faddeev formalism was extended to include core excitation [7]. This method was used to explore the role of core excitation in $(\mathrm{d}, \mathrm{p})$ reactions, and understand the origin of the dynamical effects [3]. Based on the particle-rotor model of $n+{ }^{10} \mathrm{Be}$, a number of ${ }^{11} \mathrm{Be}-$ like models were generated with a range of separation energies, while maintaining a significant coreexcited component. We used the $n+{ }^{10} \mathrm{Be}$ system as a toy model, included the first two states of ${ }^{10} \mathrm{Be}$ and coupled the additional neutron to a total spin $J=\frac{1}{2}$ just as in ${ }^{11} \mathrm{Be}$ (g.s.), but then adjusted the interaction to produce a number of models with different separation energies ranging $S_{n}=0.1-5.0$ $\mathrm{MeV}$. We then applied the method of [7], which includes dynamical core excitation in the reaction mechanism to all orders, to the ${ }^{10} \mathrm{Be}(\mathrm{d}, \mathrm{p}){ }^{11} \mathrm{Be}$ like reactions, and studied the excitation effects for beam energies from the range $E_{d}=15-90 \mathrm{MeV}$ [3]. We compared the resulting angular distributions and the spectroscopic factor that would be extracted from the cross sections, when including dynamical core excitation in the reaction model $\left(S^{f a d d}\right)$, to that of the original structure model $S^{\text {th }}$. We introduced the ratio $R_{x}=\frac{S^{\text {fadd }}}{S^{\text {th }}}$, whereby a deviation from $R_{x}=1$ provides a measure of the importance of the dynamical excitation effects in the reaction. Fig. 1 shows the behavior of $R_{x}$ as a function of beam energy for ${ }^{11} \mathrm{Be}$-like final states with several binding energies. Our results in [3] show a strong beamenergy dependence of the extracted spectroscopic factor. In fact, for intermediate beam energies 


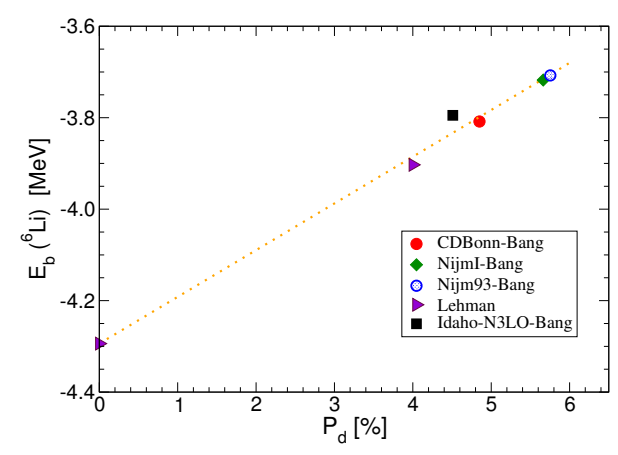

Figure 2. Three-body binding energy of ${ }^{6} \mathrm{Li}$ as a function of the deuteron d-state probability for a variety of NN interactions.

dynamical effects can be so large that the spectroscopic factor extracted by taking the ratio of data and theory is reduced to half the value of the spectroscopic factor in the original structure model. This strong dependence is most important for loosely bound systems. Given that the predicted effect is so large, it should be possible, through dedicated experiments, to test it experimentally.

\section{Toward a Faddeev theory for $(d, p)$ on heavy nuclei}

The Faddeev method of [7] cannot be applied to intermediate and heavy mass targets due to the complications that arise with screening the Coulomb interaction. Mukhamedzhanov et al. [8] rewrote the Faddeev equations in the Coulomb basis using separable interactions. Separable forms for the optical potentials were developed ([9] and references therein). Recently, the three-body problem with separable interactions was implemented and applied to ${ }^{6} \mathrm{Li}[10]$. The main conclusion in that work is that separable interactions can reproduce the exact solutions to very high accuracy. The next step in this work is to generalize the equation for positive energies, and consider elastic, breakup and rearrangement channels in detail. This work in ongoing.

As a sideline, we also explored the dependence on the bound state properties on the choice of the NN interaction. In Fig.2 the symbols show the results obtained for the three-body binding energy of ${ }^{6} \mathrm{Li}$ for a variety of $\mathrm{NN}$ interactions commonly used in our field, as a function of their prediction for the probability of the deuteron d-state. Note that only the $\mathrm{NN}$ interaction is varied, the ${ }^{4} \mathrm{He}-\mathrm{N}$ system is kept fixed with the parameters described in Ref.[10]. The dashed line serves to guide the eye and suggests that there is a linear correlation between the three-body binding energy and the deuteron d-state probability. It appears that the three-body binding energy is only sensitive to longrange properties of the $\mathrm{NN}$ interaction. Further investigation is needed to determine universality in the system.

\section{Nucleon optical potentials and the effects of nonlocality}

An essential input to nuclear reaction theories for $(\mathrm{d}, \mathrm{p})$ is the nucleon optical potential. Although in the past, nucleon optical potentials have been taken as local interactions, we understand they should be non-local and that non-locality has an important effect on reaction observables (e.g. [11, 12]). Recently we derived this potential from ab-initio coupled cluster theory. The method starts with oneparticle Green's functions, and then inverts the Dyson equation to obtain the self energy, identified with the optical potential [13]. The microscopic optical potentials obtained lacked absorption but the 


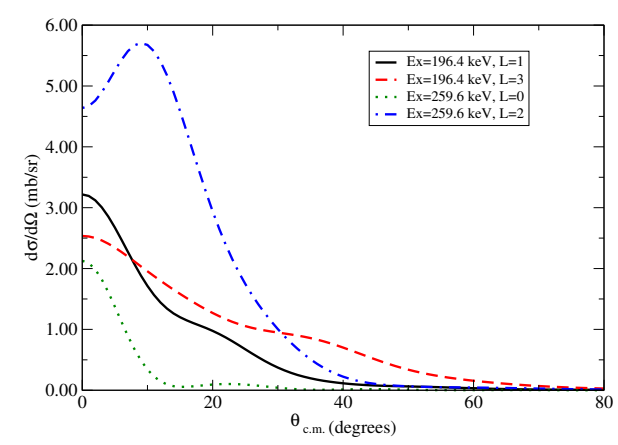

Figure 3. Angular distributions for ${ }^{30} \mathrm{P}(\mathrm{d}, \mathrm{n}){ }^{31} \mathrm{~S}$ at $30 \mathrm{MeV} / \mathrm{u}$ populating a couple of final states: dependence on the angular momentum transferred.

real part exhibited robust properties, including strong non-locality with a shape that deviates from Gaussian and varies with the position inside the nucleus. In addition, the potential generated in [13] is strongly energy dependent.

While an ab-initio approach cannot currently be directly applied to the interpretation of data, it can provide guidance to phenomenological approaches. There are two nonlocal global optical potentials available and both are energy independent. The ab-initio results call for both, nonlocality and energy dependence in the optical potential. One obvious shortcoming of the Perey and Buck potential [14] is that it was only fit to neutrons on $\mathrm{Pb}$ at low energies and does not account well for elastic scattering at $E>20 \mathrm{MeV}$. As a preliminary test of whether elastic scattering data calls for an explicit energy dependence in the non-local optical potential, we have started to investigate the problem using the standard $\chi^{2}$ minimization techniques [15]. This study shows that a new nonlocal phenomenological optical potential with a linear energy dependence can provide an overall $\chi^{2}$ that is significantly smaller than the one obtained when using the energy independent Perey and Buck potential [14], for data in the energy range $E=10-50 \mathrm{MeV}$. While promising, this problem needs to be revisited using a wider range of data and a more comprehensive parameter space.

\section{Transfer reactions to the continuum}

Several applications to astrophysics require transfer to the continuum. An example is the analysis of ${ }^{30} \mathrm{P}(\mathrm{d}, \mathrm{n}){ }^{31} \mathrm{~S}$ data to extract the proton capture on ${ }^{30} \mathrm{P}$ and inform abundance predictions for novae ejecta [16]. Because those measurements contain only integrated transfer cross sections, it was difficult to match the states predicted by shell model with those measured by GRETINA. In order to improve this analysis, it is desirable to measure the neutron angular distributions.

Transfer to the continuum is also important on the neutron dripline. One can use (d,p) reactions to populate the same continuum compound states as the $(\mathrm{n}, \gamma)$ reaction. This indirect approach requires an understanding of how probable it is to populate these compound states, as opposed to breakup. A recent review of the efforts in this area [17] discusses three independent implementations of the reaction formalism, all relying on a two step approach: first the deuteron breaks up in the field of the target, and then the neutron either gets captured (non-elastic) or flies away (elastic breakup). In [17] the three methods are benchmarked and applied to (d,p) reactions on Ca isotopes. As one moves toward the neutron dripline, the elastic breakup component increases but even for well bound systems this component is never negligible, and needs to be appropriately subtracted from the measured (d,p) cross section for a meaningful connection to $(\mathrm{n}, \gamma)$. 


\section{Concluding remarks}

In these proceedings, we discuss a number of recent developments in the field of reaction theory, with particular emphasis on nucleon and deuteron induced reactions. Recent Faddeev results for (d,p) on a light target show that core excitation is important for single-nucleon transfer, and the magnitude of the effect is strongly dependent on the beam energy. This theoretical prediction calls for dedicated experiments. We also report on progress made toward a full Faddeev approach to (d,p) reactions on heavy nuclei using separable interactions and the Coulomb basis. The results for the ${ }^{6} \mathrm{Li}$ bound state demonstrate that separable representations of realistic two-body potentials can reproduce the exact solution for the three-body bound state problem with high precision. Work on the extension to positive energy is ongoing.

In the past we found that nonlocality is important in (d,p) reactions, affecting not only the magnitude of angular distributions but also the shape. We discuss recent results in extracting an ab-initio optical potential from coupled cluster theory as well as a phenomenological approach which includes both nonlocality and energy dependence in the optical potential. Finally, a few important developments concerning transfer to the continuum are also presented in connection to extracting neutron and proton capture rates on exotic nuclei. Theories for predicting cross sections have recently been benchmarked and several improvements have been identified. ${ }^{1}$

\section{References}

[1] K. Riisager, Rev. Mod. Phys. 66, 1105 (1994)

[2] J.A. Tostevin, A. Gade, Phys. Rev. C 90, 057602 (2014)

[3] A. Deltuva, A. Ross, E. Norvaišas, F.M. Nunes, Phys. Rev. C 94, 044613 (2016)

[4] F.M. Nunes, A. Deltuva, Phys. Rev. C 84, 034607 (2011)

[5] N.J. Upadhyay, A. Deltuva, F.M. Nunes, Phys. Rev. C 85, 054621 (2012)

[6] N.C. Summers, F.M. Nunes, Phys. Rev. C 77, 049901 (2008)

[7] A. Deltuva, Phys. Rev. C 92, 064613 (2015)

[8] A. Mukhamedzhanov, V. Eremenko, A. Sattarov, Phys.Rev. C86, 034001 (2012)

[9] L. Hlophe, C. Elster, Phys. Rev. C 95, 054617 (2017)

[10] L. Hlophe, J. Lei, C. Elster, A. Nogga, F. Nunes, Phys. Rev. C, submitted (arXiv:1710.02602) (2017)

[11] L.J. Titus, F.M. Nunes, G. Potel, Phys. Rev. C 93, 014604 (2016)

[12] A. Ross, L.J. Titus, F.M. Nunes, Phys. Rev. C 94, 014607 (2016)

[13] J. Rotureau, P. Danielewicz, G. Hagen, F.M. Nunes, T. Papenbrock, Phys. Rev. C 95, 024315 (2017)

[14] F. Perey, B. Buck, Nuclear Physics 32, 353 (1962)

[15] A. Lovell, P. Bacq, P. Capel, F. Nunes, L. Titus, Phys. Rev. C, in press (2017)

[16] A. Kankainen, P. Woods, H. Schatz, T. Poxon-Pearson, D. Doherty, V. Bader, T. Baugher, D. Bazin, B. Brown, J. Browne et al., Physics Letters B 769, 549 (2017)

[17] G. Potel, G. Perdikakis, B.V. Carlson, M.C. Atkinson, W.H. Dickhoff, J.E. Escher, M.S. Hussein, J. Lei, W. Li, A.O. Macchiavelli et al., The European Physical Journal A 53, 178 (2017)

\footnotetext{
${ }^{1}$ This work was supported by National Science Foundation under Grants No. NSF-PHY-1403906, NSF-PHY-1520929 and NSF-PHY-1520972, the Stewardship Science Graduate Fellowship program under Grant No. DE-NA0002135, and the Department of Energy under Contract No. DE-FG52-08NA28552. P. C. acknowledges the support of the Deutsche Forschungsgesellschaft (DFG) with the Collaborative Research Center 1245. Ch.E. acknowledges the U. S. Department of Energy under contract No. DE-FG02-93ER40756 with Ohio University.
} 